\title{
An ME-PC Enhanced HDMR Method for Efficient Statistical Analysis of Multiconductor Transmission Line Networks
}

\author{
Abdulkadir C. Yücel, Hakan Bağcı, Senior Member, IEEE, and Eric Michielssen, Fellow, IEEE
}

\begin{abstract}
An efficient method for statistically characterizing multiconductor transmission line (MTL) networks subject to a large number of manufacturing uncertainties is presented. The proposed method achieves its efficiency by leveraging a high-dimensional model representation (HDMR) technique that approximates observables (quantities of interest in MTL networks, such as voltages/currents on mission-critical circuits) in terms of iteratively constructed component functions of only the most significant random variables (parameters that characterize the uncertainties in MTL networks, such as conductor locations and widths, and lumped element values). The efficiency of the proposed scheme is further increased using a multielement probabilistic collocation (ME-PC) method to compute the component functions of the HDMR. The ME-PC method makes use of generalized polynomial chaos (gPC) expansions to approximate the component functions, where the expansion coefficients are expressed in terms of integrals of the observable over the random domain. These integrals are numerically evaluated and the observable values at the quadrature/collocation points are computed using a fast deterministic simulator. The proposed method is capable of producing accurate statistical information pertinent to an observable that is rapidly varying across a high-dimensional random domain at a computational cost that is significantly lower than that of gPC or Monte Carlo methods. The applicability, efficiency, and accuracy of the method are demonstrated via statistical characterization of frequencydomain voltages in parallel wire, interconnect, and antenna corporate feed networks.
\end{abstract}

Index Terms-Crosstalk, generalized polynomial chaos (gPC), global sensitivity analysis, high-dimensional model representation (HDMR), interconnects, multiconductor transmission lines (MTLs), multielement probabilistic collocation (ME-PC) method, stochastic analysis, surrogate model, tolerance analysis, uncertainty quantification.

\section{INTRODUCTION}

A DVANCES in numerical algorithms and stochastic analysis techniques coupled with increases in the speed

Manuscript received October 31, 2014; revised March 8, 2015; accepted April 12, 2015. This work was supported in part by the Air Force Office of Scientific Research under Grant FA9550-10-1-0180 and in part by the Office of Naval Research under Grant N00014-11-1-0720. Recommended for publication by Associate Editor E.-P. Li upon evaluation of reviewers' comments.

A. C. Yücel and E. Michielssen are with the Department of Electrical Engineering and Computer Science, University of Michigan, Ann Arbor, MI 48109 USA (e-mail: acyucel@umich.edu; emichiel@umich.edu).

H. Bağcl is with the Division of Computer, Electrical, and Mathematical Sciences and Engineering and the Center for Uncertainty Quantification in Computational Sciences and Engineering, King Abdullah University of Science and Technology, Jeddah 23955-6900, Saudi Arabia (e-mail: hakan.bagci@kaust.edu.sa).

Color versions of one or more of the figures in this paper are available online at http://ieeexplore.ieee.org.

Digital Object Identifier 10.1109/TCPMT.2015.2424679 and memory capacity of computers recently have enabled the statistical characterization of complex multiconductor transmission line (MTL) networks. Methods developed for this purpose provide statistics of observables (such as voltages and currents on mission-critical circuits and components), given uncertainties due to manufacturing tolerances and processinduced variability (such as values of lumped electronic components, the constitutive parameters of substrates, and the positions and widths of interconnects), and excitations. These statistics, which are encapsulated by the means, standard deviations, probability density functions (PDFs), and sensitivity indices of the observables in MTL networks, are used for quantifying a system's expected performance, failure rate, and operational margins of error.

The effects of uncertainties parameterized by random variables in MTL networks have traditionally been quantified through adjoint sensitivity methods [1], analytical probabilistic models [2], and Monte Carlo (MC) techniques [3], [4]. Among these methods, MC techniques are the most easily applied to complex MTL networks. Classical MC methods use a deterministic MTL simulator to compute values of observables for many realizations of the network, which are constructed by sampling the assumed/known PDFs of the random variables. MC methods are easily implemented and readily provide the statistics of all observables. That said, they usually converge slowly [5] and hence may call for many executions of the MTL simulator to provide reasonably accurate results.

Stochastic Galerkin/collocation schemes leveraging the generalized polynomial chaos (gPC) expansion address the convergence concerns of MC methods. Recently, they have been deployed successfully in computational electromagnetic analysis [6]-[16]. gPC expansion-based methods construct a highly accurate and efficient-to-compute surrogate model of the observable in terms of entire-domain orthogonal polynomials. This surrogate model is then used to obtain the observable's mean, standard deviation, and sensitivity indices, or probed by an MC method that provides its PDF. Coefficients of the polynomial expansion are computed using Galerkin or collocation methods [17]. While gPC Galerkin methods provide slightly more accurate statistics than their collocation counterparts [17], they require rather intrusive modifications to the underlying deterministic MTL simulator [18]-[23]. gPC collocation methods only call for the evaluation of integrals involving the observable over the domain of random variables to compute the polynomial expansion coefficients and construct the surrogate model [17]. These integrals are evaluated using efficient multidimensional integral rules [17], 
where the observable values at integration (collocation) points are computed using an existing deterministic MTL simulator [24]-[28]. Consequently, gPC collocation methods are nonintrusive and computationally cheaper than their Galerkin counterparts [17]. gPC Galerkin and collocation methods converge rapidly for an observable that varies smoothly across the random domain [17]. However, they become inefficient and inaccurate when the observable exhibits rapid variations and/or discontinuities [29], [30].

Multielement gPC and probabilistic collocation (ME-PC) schemes have been developed to address this shortcoming of the gPC Galerkin and collocation methods, respectively [29], [30]. These schemes achieve their accuracy and efficiency via $h$-adaptive refinement techniques, i.e., by adaptively and recursively dividing the random domain into subdomains and generating a (separate) local gPC expansion on each of them. Even with this capability, gPC-based methods and their accelerated versions remain inefficient for surrogate model generation in high-dimensional random domains. This deficiency stems from the fact that the number of orthogonal polynomials used in the gPC expansion(s) scales exponentially with the number of random variables [31], [32]. Similar deficiencies are oftentimes encountered in macromodeling [33] and model-order reduction techniques (see the references in [34]) as well. To address this limitation, semi-intrusive stochastic macromodeling techniques [35], [36] and a nonintrusive dimension adaptive sparse grid method [37] have been used in the context of stochastic MTL analysis; implementation of the former hinges on access to the deterministic simulator's inner workings while the latter is easily coupled with any deterministic simulator. In parallel, ME-PC methods have recently been hybridized with high-dimensional model representation (HDMR) techniques [31]. These techniques allow for the generation of a surrogate model in a highdimensional random domain by bootstrapping surrogate models generated in low-dimensional random domains that are carefully selected subsets of the initial random domain.

This paper elucidates a computational framework for statistically characterizing observables in MTL networks, which (potentially) vary rapidly throughout high-dimensional random domains. The proposed framework leverages an HDMR technique to generate the surrogate model of the observable in terms of finite sums of component functions, which represent the individual and combined contributions of the random variables to the observable [38]. The HDMR is constructed iteratively by only including component functions pertinent to the most important random variables, and therefore dramatically reduces the cost of surrogate model construction/computation [32]. The component functions included in the HDMR are computed using the ME-PC method [30], [39], [40], which tailors the distribution of the collocation/integration points in the random domain depending on the variation of the observable. The observable values at these collocation/integration points can be computed using any desired deterministic MTL simulator; here, a fast integral equation-based MTL [41] and IE3D full-wave [42] simulators are used for this purpose. Furthermore, the proposed framework combines the HDMR with the partial fraction expansion to generate a surrogate model of the observable over a wide frequency band [43]-[45]. Upon generation of the accurate surrogate model, the classical MC method is used to compute the statistics of the observable while accounting for the PDFs of the random variables. The accuracy and efficiency of the proposed framework are demonstrated via its application to the statistical characterization of crosstalkinduced voltages in parallel wire and interconnect networks, and voltages across the terminals of a corporate feed network of a linear patch antenna array.

\section{FORMULATION}

\section{A. Mathematical Description of the Problem}

Let $x_{i}, i=1, \ldots, N_{\mathrm{dof}}$, represent $N_{\text {dof }}$ mutually independent random variables, each of which parameterizes one uncertainty in the MTL network. Each random variable $x_{i}$ is distributed with an assumed/known PDF $\xi_{i}(\cdot)$ defined in a finite 1-D random domain $D_{i}=\left[a_{i}, b_{i}\right]$. For the compactness of the formulation, let $\mathbf{x}$ denote an $N_{\text {dof }}$-dimensional random vector with entries, $x_{i}, i=1, \ldots, N_{\text {dof }}$, i.e., $\mathbf{x}=\left[x_{1}, x_{2}, \ldots, x_{N_{\text {dof }}}\right]$, defined in the random domain $D=\prod_{i=1}^{N_{\text {dof }}} D_{i}$.

Let $V(\mathbf{x})$ denote an observable in the MTL network; $V(\mathbf{x})$ is typically a complicated and nonlinear function of $\mathbf{x}$ and can only be evaluated using a deterministic MTL simulator. In realistic models of complex MTL networks, oftentimes $N_{\text {dof }}$ is large. In addition, nonlinear dependence on $\mathbf{x}$ when combined with electromagnetic resonances might render $V(\mathbf{x})$ a very rapidly varying function of $\mathbf{x}$. Consequently, classical PC methods, which make use of gPC expansion to a generate surrogate model of $V(\mathbf{x})$, become highly inefficient and/or inaccurate [40].

To address these inefficiency and inaccuracy problems, the proposed method uses the following.

1) An HDMR technique to generate an accurate surrogate model of $V(\mathbf{x})$, in terms of efficient-to-compute component functions in only the most significant random variables [32], [38] (Sections II-B and II-C).

2) The ME-PC scheme to compute each of these component functions that are defined over lower dimensional domains but vary rapidly across them [30], [31] (Section II-D).

Upon generation of an accurate surrogate model, it is probed via MC method that takes into account the PDFs $\xi_{i}(\cdot) \quad\left(i=1, \ldots, N_{\text {dof }}\right)$ to obtain the statistics of $V(\mathbf{x})$.

\section{B. HDMR Technique}

The HDMR technique decomposes the $N_{\text {dof }}$-variate $V(\mathbf{x})$ into component functions defined over lower dimensional domains (i.e., random domains of subsets of $\mathbf{x}$ ). While iteratively building the HDMR, only the most significant component functions are retained to minimize the cost of surrogate model construction/computation. This iterative construction is detailed next. The HDMR of $V(\mathbf{x})$ reads

$$
V(\mathbf{x})=\sum_{\mathbf{u} \subseteq \Omega} F_{\mathbf{u}}\left(\mathbf{x}_{\mathbf{u}}\right)
$$


where $\Omega=\left\{1, \ldots, N_{\mathrm{dof}}\right\}$ is the set of random variable indices, $\mathbf{u}$ is a subset of $\Omega$, i.e., $\mathbf{u} \subseteq \Omega$, and $|\mathbf{u}|$ is the cardinality of $\mathbf{u}$. Moreover, $\mathbf{x}_{\mathbf{u}}$ denotes a $|\mathbf{u}|$-dimensional random vector and $F_{\mathbf{u}}\left(\mathbf{x}_{\mathbf{u}}\right)$ represents a component function defined over $D$. As an example, for $\mathbf{u}=\emptyset, F_{\mathbf{u}}\left(\mathbf{x}_{\mathbf{u}}\right)=F_{\varnothing}\left(x_{\varnothing}\right)=F_{0}$ is the zerothorder component function; it is constant over $D$. For $\mathbf{u}=\{1\}$, $F_{\mathbf{u}}\left(\mathbf{x}_{\mathbf{u}}\right)=F_{1}\left(x_{1}\right)$ is the first-order component function that describes the contribution of $x_{1}$ to $V(\mathbf{x})$. For $\mathbf{u}=\{1,2,3\}$, $F_{\mathbf{u}}\left(\mathbf{x}_{\mathbf{u}}\right)=F_{123}\left(x_{1}, x_{2}, x_{3}\right)$ is the third-order component function that represents the combined impact of $x_{1}, x_{2}$, and $x_{3}$ on $V(\mathbf{x})$. The role of component functions in the HDMR expansion is best illustrated by an example. Assume that $V(\mathbf{x})$ is a function of three random variables $\left(N_{\text {dof }}=3\right)$ with indices $\Omega=\{1,2,3\}$. In the HDMR of $V(\mathbf{x})$, the component functions $F_{\mathbf{u}}\left(\mathbf{x}_{\mathbf{u}}\right)$ corresponding to all possible subsets $\mathbf{u}$ of $\Omega$ are

$\begin{array}{lll}F_{0}, & \mathbf{u}=\emptyset, & |\mathbf{u}|=0 \\ F_{1}\left(x_{1}\right), & \mathbf{u}=\{1\}, & \mid \mathbf{u}=1 \\ F_{2}\left(x_{2}\right), & \mathbf{u}=\{2\}, & |\mathbf{u}|=1 \\ F_{3}\left(x_{3}\right), & \mathbf{u}=\{3\}, & |\mathbf{u}|=1 \\ F_{12}\left(x_{1}, x_{2}\right), & \mathbf{u}=\{1,2\}, & |\mathbf{u}|=2 \\ F_{13}\left(x_{1}, x_{3}\right), & \mathbf{u}=\{1,3\}, & |\mathbf{u}|=2 \\ F_{23}\left(x_{2}, x_{3}\right), & \mathbf{u}=\{2,3\}, & |\mathbf{u}|=2 \\ F_{123}\left(x_{1}, x_{2}, x_{3}\right), & \mathbf{u}=\{1,2,3\}, & |\mathbf{u}|=3 .\end{array}$

If all component functions in (2) are retained in the HDMR (1), then $V(\mathbf{x})$ is expressed as

$$
\begin{aligned}
V(\mathbf{x})= & F_{0}+F_{1}\left(x_{1}\right)+F_{2}\left(x_{2}\right)+F_{3}\left(x_{3}\right)+F_{12}\left(x_{1}, x_{2}\right) \\
& +F_{13}\left(x_{1}, x_{3}\right)+F_{23}\left(x_{2}, x_{3}\right)+F_{123}\left(x_{1}, x_{2}, x_{3}\right) .
\end{aligned}
$$

The advantage of leveraging HDMR for surrogate model generation is illustrated on an observable that consists of only monomials, i.e., $V(\mathbf{x})=x_{1}^{3}+x_{2}^{3}+x_{3}^{3}$. It is clear that for this simple observable, only the component functions $F_{1}\left(x_{1}\right)$, $F_{2}\left(x_{2}\right)$, and $F_{3}\left(x_{3}\right)$ are called for and the remaining functions can be omitted without any loss of accuracy in expansion (3). In many real-world problems, physical observables can be approximated by low-order component functions [31], which makes the HDMRs highly efficient in constructing surrogate models of observables originally defined in high-dimensional random domains.

The component functions $F_{\mathbf{u}}\left(\mathbf{x}_{\mathbf{u}}\right), \mathbf{u} \subseteq \Omega$, can be obtained by the analysis of variance HDMR and CUT-HDMR techniques. The former oftentimes is not suitable for surrogate model generation in high-dimensional random domains [32] and hence not considered further. The CUT-HDMR technique generates $F_{\mathbf{u}}\left(\mathbf{x}_{\mathbf{u}}\right)$ from observable values on lines, planes, and hyperplanes, i.e., cuts that pass through a reference point, $\overline{\mathbf{x}}$, in $D$, i.e.

$$
F_{\mathbf{u}}\left(\mathbf{x}_{\mathbf{u}}\right)=\left.V(\mathbf{x})\right|_{\mathbf{x}=\overline{\mathbf{x}} \backslash \mathbf{x}_{\mathbf{u}}}-\sum_{\mathbf{v} \subset \mathbf{u}} F_{\mathbf{v}}\left(\mathbf{x}_{\mathbf{v}}\right) .
$$

Here, $\mathbf{x}=\overline{\mathbf{x}} \backslash \mathbf{x}_{\mathbf{u}}$ indicates that random variables with indices not belonging to $\mathbf{u}$ are set to their corresponding values at the reference point $\overline{\mathbf{x}}$, which is typically selected as the center of mass of the random domain, i.e., $\overline{\mathbf{x}}=$ $\left[\bar{x}_{1}, \ldots, \bar{x}_{N_{\mathrm{dof}}}\right]=\left[\left(a_{1}+b_{1}\right) / 2, \ldots,\left(a_{N_{\mathrm{dof}}}+b_{N_{\mathrm{dof}}}\right) / 2\right]$. The CUT-HDMR technique constructs the component functions in expansion (3) using

$$
\begin{aligned}
& F_{0}=V(\overline{\mathbf{x}}), \quad \quad \mathbf{u}=\varnothing \\
& F_{1}\left(x_{1}\right)=V\left(x_{1}, \bar{x}_{2}, \bar{x}_{3}\right)-F_{0}, \quad \mathbf{u}=\{1\} \\
& F_{2}\left(x_{2}\right)=V\left(\bar{x}_{1}, x_{2}, \bar{x}_{3}\right)-F_{0}, \quad \mathbf{u}=\{2\} \\
& F_{3}\left(x_{3}\right)=V\left(\bar{x}_{1}, \bar{x}_{2}, x_{3}\right)-F_{0}, \quad \mathbf{u}=\{3\} \\
& F_{12}\left(x_{1}, x_{2}\right)=V\left(x_{1}, x_{2}, \bar{x}_{3}\right)-F_{0} \\
& -F_{1}\left(x_{1}\right)-F_{2}\left(x_{2}\right), \quad \mathbf{u}=\{1,2\} \\
& F_{13}\left(x_{1}, x_{3}\right)=V\left(x_{1}, \bar{x}_{2}, x_{3}\right)-F_{0} \\
& -F_{1}\left(x_{1}\right)-F_{3}\left(x_{3}\right), \quad \mathbf{u}=\{1,3\} \\
& F_{23}\left(x_{2}, x_{3}\right)=V\left(\bar{x}_{1}, x_{2}, x_{3}\right)-F_{0} \\
& -F_{2}\left(x_{2}\right)-F_{3}\left(x_{3}\right), \quad \mathbf{u}=\{2,3\} \\
& F_{123}\left(x_{1}, x_{2}, x_{3}\right)=V\left(x_{1}, x_{2}, x_{3}\right)-F_{12}\left(x_{1}, x_{2}\right) \\
& -F_{13}\left(x_{1}, x_{3}\right)-F_{23}\left(x_{2}, x_{3}\right) \\
& -F_{1}\left(x_{1}\right)-F_{2}\left(x_{2}\right)-F_{3}\left(x_{3}\right)-F_{0} \text {, } \\
& \mathbf{u}=\{1,2,3\} \text {. }
\end{aligned}
$$

The most efficient scheme to generate the surrogate model of $V(\mathbf{x})$ by CUT-HDMR technique is to interpolate $F_{\mathbf{u}}\left(\mathbf{x}_{\mathbf{u}}\right)$ directly from the component function with the highest cardinality. However, this method is inefficient if the significant/unneeded component functions are not known a priori. Another scheme is to start from the component function with the lowest cardinality and recursively construct $F_{\mathbf{u}}\left(\mathbf{x}_{\mathbf{u}}\right)$ as the cardinality is increased. This scheme calls for the computation of the observable value at $\overline{\mathbf{x}}$ first to obtain the zeroth-order component function. Next, observable values are computed on lines, planes, and hyperplanes passing through $\overline{\mathbf{x}}$ (i.e., $\mathbf{x}=\overline{\mathbf{x}} \backslash \mathbf{x}_{\mathbf{u}}$ ) to construct first-order, second-order, and higher order component functions, respectively. The computation of the observable values (and the component functions) called for by this recursive scheme can be accelerated using a multivariate interpolator. In this paper, error-controllable ME-PC method [30], [31], [39], [40] is used for this purpose, since the observable is a function with smooth and fast variations and/or discontinuities (Section II-D). In addition, the number of component functions in expansion (3) can be reduced significantly by eliminating those that do not contribute to the observable. This is carried out using an iterative scheme, as described in Section II-C.

\section{Iterative HDMR Technique}

The number of component functions in the HDMR scales as $\sum_{j=0}^{|\mathbf{u}|} N_{\mathrm{dof}} ! /\left(j !\left(N_{\mathrm{dof}}-j\right) !\right)$, which prohibits its use in generating surrogate models in high-dimensional domains, i.e., when $N_{\text {dof }}$ is large. This limitation can be overcome using an iterative scheme that automatically selects random variables with significant impact on $V(\mathbf{x})$ and only includes the higher order component functions pertinent to these variables in the HDMR. The iterative scheme first computes the weights associated with the first-order component functions as [32]

$$
\Upsilon_{\mathbf{u}}=\left|E\left[F_{\mathbf{u}}\left(\mathbf{x}_{\mathbf{u}}\right)\right] / F_{0}\right| ; \quad|\mathbf{u}|=1 .
$$

The weights $\Upsilon_{\mathbf{u}}$ quantify the contributions of the first-order component functions' means, $E\left[F_{\mathbf{u}}\left(\mathbf{x}_{\mathbf{u}}\right)\right]=\int F_{\mathbf{u}}\left(\mathbf{x}_{\mathbf{u}}\right) d \mathbf{x}_{\mathbf{u}}$, 
to the mean of $V(\mathbf{x})$ computed at the zeroth level, $F_{0}$. If $\Upsilon_{\mathbf{u}}$, $|\mathbf{u}|=1$, exceeds a prescribed tolerance $\varepsilon_{1}$, then the component function is assumed to contribute significantly to $V(\mathbf{x})$ and the pertinent random variable is identified as significant. The indices of the significant random variables are stored in the index set $\Omega$. Next, the second-order component functions pertinent to these significant random variables are considered as candidates for constructing the second level HDMR. Component functions are included in the expansion only if their weights computed using [32]

$$
\Upsilon_{\mathbf{u}}=\left|E\left[F_{\mathbf{u}}\left(\mathbf{x}_{\mathbf{u}}\right)\right]\right| /\left|\sum_{|\mathbf{v}|<|\mathbf{u}|-1} E\left[F_{\mathbf{v}}\left(\mathbf{x}_{\mathbf{v}}\right)\right]\right| ; \quad|\mathbf{u}| \geq 2
$$

exceed $\varepsilon_{1}$. This selection/elimination process is continued iteratively as the level is increased until the HDMR is assumed to have converged within a prescribed tolerance. Convergence criterion is defined as $\varepsilon_{2}>\kappa$, where $\kappa$ is the decay rate of the relative difference between observable means computed at two consecutive levels [32]

$$
\kappa=\frac{\left|\sum_{|\mathbf{v}|<|\mathbf{u}|} E\left[F_{\mathbf{v}}\left(\mathbf{x}_{\mathbf{v}}\right)\right]-\sum_{|\mathbf{v}|<|\mathbf{u}|-1} E\left[F_{\mathbf{v}}\left(\mathbf{x}_{\mathbf{v}}\right)\right]\right|}{\left|\sum_{|\mathbf{v}|<|\mathbf{u}|-1} E\left[F_{\mathbf{v}}\left(\mathbf{x}_{\mathbf{v}}\right)\right]\right|} .
$$

Upon completion of the HDMR construction, the component functions identified as insignificant are also included in the expansion to increase the accuracy of surrogate model as they are already computed during the iterative construction. It should be noted here that if multiple observables' surrogate models are being constructed simultaneously, $\Upsilon_{\mathbf{u}}$ and $\kappa$ are computed for all observables separately. If only one of the $\Upsilon_{\mathbf{u}}$ computed for all observables exceeds $\varepsilon_{1}$, then the component function pertinent to $\Upsilon_{\mathbf{u}}$ is identified as a significant component function. Likewise, if all of the $\kappa$ computed for observables is smaller than $\varepsilon_{2}$, then the HDMRs generated for all observables are assumed to have converged.

The iterative procedure described above can be better explained by an example illustrated with a flowchart of the procedure (Fig. 1). In this example $\left(N_{\mathrm{dof}}=5\right)$, the procedure is initialized by storing the indices of the zeroth- and first-order component functions in the index set $\Omega$ and computing $F_{0}$. Next, the iterative construction is continued until $\kappa \leq \varepsilon_{2}$ or $\Omega$ reverts to the null set (Fig. 1). Assume that, after computing $F_{\mathbf{u}}\left(\mathbf{x}_{\mathbf{u}}\right),|\mathbf{u}|=1$, via the ME-PC method (Section II-D) and $\Upsilon_{\mathbf{u}},|\mathbf{u}|=1$, using (6), and comparing $\Upsilon_{\mathbf{u}}$ to $\varepsilon_{1}$, only the random variables (or the first-order component functions) with indices $\{2,4,5\}$ are found to be significant at level one (Fig. 1). Then, the indices of the candidate component functions, $\{2,4\},\{2,5\}$, and $\{4,5\}$, to be considered at level two are stored in $\Omega$. In the second iteration for level two, the $\Upsilon_{\mathbf{u}}$, $|\mathbf{u}|=2$, are computed using (7) only for the second-order component functions $F_{24}\left(x_{2}, x_{4}\right), F_{25}\left(x_{2}, x_{5}\right)$, and $F_{45}\left(x_{4}, x_{5}\right)$ (those represent the combined effect of the significant random variables with indices $\{2,4,5\})$ and compared with $\varepsilon_{1}$. If any of these component functions is found to be insignificant (i.e., $\left.\Upsilon_{\mathbf{u}}<\varepsilon_{1}\right)\left[F_{45}\left(x_{4}, x_{5}\right)\right.$ in this example], then no third-order component function is considered for inclusion in the HDMR as index set $\Omega$ is empty. On the other hand, if all three component functions $F_{24}\left(x_{2}, x_{4}\right), F_{25}\left(x_{2}, x_{5}\right)$, and $F_{45}\left(x_{4}, x_{5}\right)$ are found to be significant, then the thirdorder component function $F_{245}\left(x_{2}, x_{4}, x_{5}\right)$ is considered for



Fig. 1. Flow chart of the iterative HDMR algorithm as well as its execution for an example with $N_{\text {dof }}=5$.

inclusion in the third level of HDMR. Note that $\kappa$ is computed using (8) at the end of each iteration; if $\kappa \leq \varepsilon_{2}$, then the HDMR is assumed to have converged and iterations are terminated. At the end of the iterative process, the HDMR is constructed by the component functions already computed via the ME-PC method, the indices of which are stored in index set $\Omega^{\prime}$ (Fig. 1).

Upon generation of the surrogate model, its statistical moments [approximating those of $V(\mathbf{x})$ ] can be obtained by sampling it with an MC method that accounts for the assumed PDFs of the random variables, $\xi_{i}(\cdot), i=1, \ldots, N_{\mathrm{dof}}$. However, an approximation to the mean of $V(\mathbf{x})$ can directly be calculated by [32]

$$
E[V(\mathbf{x})]=\sum_{\mathbf{u} \subseteq \Omega} E\left[F_{\mathbf{u}}\left(\mathbf{x}_{\mathbf{u}}\right)\right] .
$$

Likewise, the variance of $V(\mathbf{x})$ can be computed using [32]

$$
\operatorname{var}[V(\mathbf{x})]=\sum_{\mathbf{u} \subseteq \Omega} \operatorname{var}\left[F_{\mathbf{u}}\left(\mathbf{x}_{\mathbf{u}}\right)\right]
$$

where $\operatorname{var}\left[F_{\mathbf{u}}\left(\mathbf{x}_{\mathbf{u}}\right)\right]=E\left[\left(F_{\mathbf{u}}\left(\mathbf{x}_{\mathbf{u}}\right)\right)^{2}\right]-\left(E\left[F_{\mathbf{u}}\left(\mathbf{x}_{\mathbf{u}}\right)\right]\right)^{2}$. In addition, global sensitivity indices (also called Sobol indices) are computed from the ratio of each component function's variance to that of $V(\mathbf{x})$ [46], i.e.

$$
S_{\mathbf{u}}=\operatorname{var}\left[F_{\mathbf{u}}\left(\mathbf{x}_{\mathbf{u}}\right)\right] / \sum_{\mathbf{u} \subseteq \Omega} \operatorname{var}\left[F_{\mathbf{u}}\left(\mathbf{x}_{\mathbf{u}}\right)\right] .
$$

These indices quantify the contributions of random variables and their higher order correlations to the variance of the observable. 


\section{ME-PC Method}

Oftentimes, observables in complex and large MTL networks are rapidly varying functions of random variables. This prohibits an efficient use of traditional $\mathrm{gPC}$ collocation methods in constructing surrogate models. The ME-PC method accelerates these schemes by employing an $h$-adaptive refinement scheme, as detailed in [30] and [40].

Similarly, in this paper, the ME-PC scheme is used for accelerating the computation of component functions during the iterative construction of the HDMR. As described in Sections II-B and II-C, this procedure calls for the computation of the observable values on cuts (i.e., lines, planes, and hyperplanes) passing through the reference point $\overline{\mathbf{x}}$. The cost of this operation becomes prohibitive if an MTL simulator is used every time, the observable value needs to be computed.

Therefore, first-order, second-order, and higher order component functions are interpolated efficiently and accurately from the observable values on the cuts using the ME-PC method. The ME-PC method achieves its accuracy and efficiency by adaptively and recursively dividing the cuts into subelements, on each of which a separate low-order gPC polynomial expansion is constructed as an interpolant. The integrals that provide the coefficients of these expansions/interpolants are evaluated numerically using a multidimensional integration rule [47]-[49]. The values of the observable at the integration points are computed using either an integral equation-based MTL simulator [41] or IE3D full-wave simulator [42]. It is noteworthy that many integration points used to evaluate the integrals are commonly used while interpolating component functions. The observable values on these integration points can be computed using deterministic simulator only once and then obtained from a lookup table without running the deterministic simulator when needed. This yields significant reduction in number of costly deterministic simulations especially when tensorproduct-based Gauss-Legendre quadrature rules with odd number of integration points are used.

\section{E. Broadband Statistical MTL Analysis}

Typically, statistical characterization of the MTL networks requires construction of surrogate models over a broadband of frequencies. The ME-PC enhanced HDMR described in the previous sections is used to generate a compact and efficientto-compute surrogate model of the observable at a single frequency point. The efficiency of this approach is maintained in the case of broadband analysis by combining the ME-PC enhanced HMDR with a partial fraction expansion in frequency.

Let $V(\mathbf{x}, f)$ represent the observable of the broadband statistical MTL analysis and $f$ denote the frequency within the range $\left[f_{\text {beg }}, f_{\text {end }}\right]$. While ME-PC enhanced iterative HDMR is employed to generate the surrogate model of the observable in $\mathbf{x}$, a partial fraction expansion [43] is used to construct the surrogate model along $f$. Then, $V(\mathbf{x}, f)$ is expressed as

$$
V(\mathbf{x}, f) \approx \sum_{m=1}^{N_{\mathrm{pf}}} \sum_{\mathbf{u} \subseteq \Omega} F_{\mathbf{u}}\left(\mathbf{x}_{\mathbf{u}}\right) \mathrm{PF}_{m}(f) .
$$

Here, $N_{\mathrm{pf}}$ represents the number of partial fractions and $\mathrm{PF}_{m}(f)$ denotes the partial fraction defined as [43]

$$
\mathrm{PF}_{m}(f)=\frac{c_{m}}{f-a_{m}}+d+f h
$$

where $a_{m}$ and $c_{m}, m=1, \ldots, N_{\mathrm{pf}}$, are the poles and residues and $d$ and $h$ are the constants. To find the optimum poles and residues of the partial fractions and constants in (13), the wellknown vector fitting algorithm is used [50]. This algorithm minimizes the data misfit between the frequency samples of the partial fraction expansion and the surrogate model of $V(\mathbf{x}, f)$ along $\mathbf{x}$. More specifically, it calls for the computation of the surrogate model of $V(\mathbf{x}, f)$, which is constructed using the ME-PC enhanced iterative HDMR at frequency samples $f_{n}=f_{\text {beg }}+(n-1)\left(f_{\text {end }}-f_{\text {beg }}\right) /\left(N_{s}-1\right), n=1, \ldots, N_{s}$. Here, $N_{s}$ is selected large enough to maintain the lowest possible least squares approximation error in the minimization of the data misfit [43], [44]. Once the poles, residues, and constants in (13) are obtained, the expansion in (12) can be used as an accurate and efficient-to-compute surrogate model of $V(\mathbf{x}, f)$ within the frequency band [ $\left.f_{\text {beg }}, f_{\text {end }}\right]$.

\section{NUMERICAL RESULTS}

This section demonstrates the efficiency and accuracy of the proposed method via its application to the statistical characterization of voltages in various MTL networks. In all examples, $V(\mathbf{x}, f)$ and $\tilde{V}(\mathbf{x}, f)$ represent the actual observable and its surrogate model generated by the proposed ME-PC enhanced iterative HDMR technique or another nonintrusive technique, respectively. Unless stated, otherwise, the iterative HDMR technique uses $\varepsilon_{1}=10^{-2}$ and $\varepsilon_{2}=10^{-16}$ and the ME-PC method with user-defined constants $\tau_{1}=\tau_{2}=0.5$ and tolerance $\beta=10^{-2}$ approximates the observables on recursively and adaptively refined elements of cuts using $p$ th-order gPC expansions (see details pertinent to $\tau_{1}, \tau_{2}, \beta$, and $p$ in [40]). The integrals that provide the coefficients of the $p$ th-order $\mathrm{gPC}$ expansions are computed by the tensor product integration rule using specified number of GaussLegendre points in each dimension [40]. Means, standard deviations, PDFs, and sensitivity indices pertinent to $V(\mathbf{x}, f)$ and $\tilde{V}(\mathbf{x}, f), n=1, \ldots, N_{f}$, are extracted using the MC method that samples the domain $D$ based on the assumed PDFs of the random variables. In addition, in all problems, upper and lower bounds of variation in $V(\mathbf{x}, f)$ and $\tilde{V}(\mathbf{x}, f)$ are computed using the mean and standard deviation as $E[\cdot] \pm \operatorname{std}[\cdot]$. Let $\mathbf{x}_{i}, \quad i=1, \ldots, N_{\mathrm{MC}}$, represent the MC samples (i.e., random network realizations) selected due to the assumed PDFs of the random variables. The average error in the surrogate models is computed using

$$
\text { err }=\frac{1}{N_{f}} \sum_{n=1}^{N_{f}} \sqrt{\frac{\sum_{i=1}^{N_{\mathrm{MC}}}\left[\left|\tilde{V}\left(\mathbf{x}_{i}, f_{n}\right)\right|-\left|V\left(\mathbf{x}_{i}, f_{n}\right)\right|\right]^{2}}{\sum_{i=1}^{N_{\mathrm{MC}}}\left|V\left(\mathbf{x}_{i}, f_{n}\right)\right|^{2}}}
$$

Here, $f_{n}, n=1, \ldots, N_{f}$ are the frequency points equally spaced in the band $\left[f_{\text {beg }}, f_{\text {end }}\right]$. All deterministic simulations were performed on a dual hexacore X5650 Intel processor located at the Advanced Research Computing-Technology Services High Performance Computing Center at the University of Michigan. 


\section{A. Parallel Wire Network}

The proposed method is used to statistically characterize crosstalk-induced voltages in a parallel wire network [Fig. 2(a)]. The network consists of five lossless 10-m-long wires with $0.4-\mathrm{mm}$ radius. The first wire in the network is excited by a sinusoidal voltage source [Fig. 2(a)]. The wires are positioned parallel to each other above an infinite perfect electrically conducting (PEC) ground at $z=0 \mathrm{~m}$, centered at $\left(c x_{j}, 0, c z_{j}\right) \mathrm{cm}(j=1, \ldots, 5)$, and connected to resistors at the source end, $R_{S, j}(j=1, \ldots, 5)$. At the load end, the first four wires are connected to resistors $R_{L, j}(j=1, \ldots, 4)$ while the fifth wire is terminated by a circuit in either Block A or Block B [Fig. 2(a)]. Two different scenarios are considered in accordance to the termination of fifth wire.

1) Resistor Termination: In the first scenario, the fifth wire is terminated by Block $\mathrm{A}$, which consists of a resistor $R_{L, 5}$ [Fig. 2(a)]. The voltage source is operated in the $10-100-\mathrm{MHz}$ range. Twenty parameters characterize uncertainty in this network $\left(N_{\mathrm{dof}}=20\right)$. They are the positions of the wires along the $x$-direction $c x_{j}(j=1, \ldots, 5)$, the positions of the wires along the $z$-direction $c z_{j}(j=1, \ldots, 5)$, the values of the resistors at the source end $R_{S, j}(j=1, \ldots, 5)$, and the values of the resistors at the load end $R_{L, j}(j=1, \ldots, 5) ;(\mathbf{x}=$ $\left.\left[c x_{1}, \ldots, c x_{5}, c z_{1}, \ldots, c z_{5}, R_{S, 1}, \ldots, R_{S, 5}, R_{L, 1}, \ldots, R_{L, 5}\right]\right)$. All random variables are assumed normally distributed with means and standard deviations $\left(\mu_{i}, \sigma_{i}\right) \quad(i=1, \ldots, 20)$ specified in Table I. It should be noted that the random variables' means correspond to those in [24] and each normal distribution is approximated by a truncated Gaussian defined in the range $\left[a_{i}, b_{i}\right]=\left[\mu_{i}-3 \sigma_{i}, \mu_{i}+3 \sigma_{i}\right]$ [51]. The observable is the crosstalk-induced voltage across the resistor in Block A. The ME-PC enhanced iterative HDMR technique (combined with partial fraction expansion) required 353 samples of $V(\mathbf{x}, f)$ at 75 frequency points $\left(N_{s}=75\right)$ to construct the surrogate model $\tilde{V}(\mathbf{x}, f)$; the adaptive ME-PC used second-order gPC expansions, coefficients of which are obtained via tensor-product-based Gauss-Legendre quadrature rule with three points in each dimension.

The means and standard deviations of $\left|V\left(\mathbf{x}, f_{n}\right)\right|$ and $\left|\tilde{V}\left(\mathbf{x}, f_{n}\right)\right|, n=1, \ldots, N_{f}, N_{f}=1001$, are computed using an MC method with $N_{\mathrm{MC}}=25000$ samples. Fig. 2(b) plots the means, as well as the upper and lower bounds of voltage variations. Both quantities match well demonstrating the accuracy of $\tilde{V}(\mathbf{x}, f)$. MC samples of $V\left(\mathbf{x}, f_{n}\right)$ and $\tilde{V}\left(\mathbf{x}, f_{n}\right)$ are then used in (14) and the error is found to be err $=2.88 \times 10^{-3}$, again verifying the accuracy of the proposed method. It should be noted here that constructing $\tilde{V}(\mathbf{x}, f)$ and sampling it with the MC method comes with a computational cost that is just a small fraction of sampling directly $V(\mathbf{x}, f)$.

The PDFs of $\left|V\left(\mathbf{x}, f_{n}\right)\right|$ and $\left|\tilde{V}\left(\mathbf{x}, f_{n}\right)\right|$ at three frequency points (near to resonant frequencies) are extracted by binning the samples obtained in the MC simulations [Fig. 2(c)-(e)]. The PDFs of $|\tilde{V}(\mathbf{x}, f)|$ are nearly identical to those of $|V(\mathbf{x}, f)|$. In addition, global sensitivity indices of the random variables are computed using $\tilde{V}(\mathbf{x}, f)$ [Fig. 2(f)] [52]. Clearly, uncertainties in the distances between the wires and ground plane, $c z_{j}(j=1, \ldots, 5)$, the positions of the
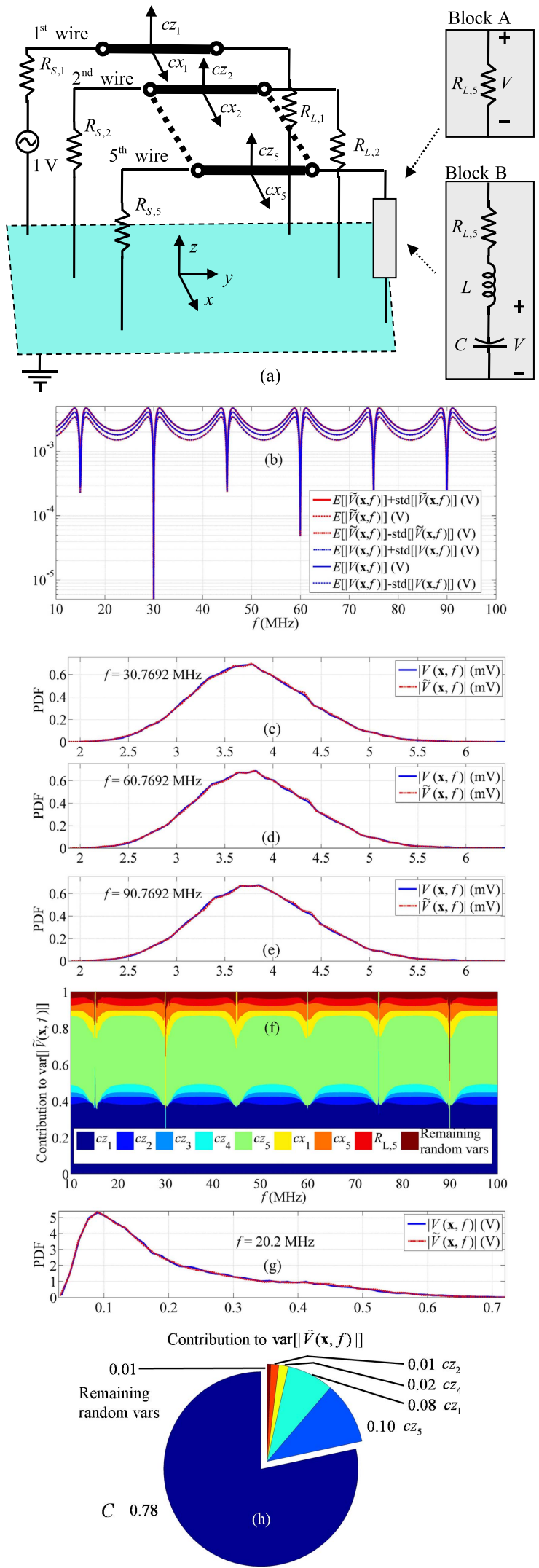

Fig. 2. (a) Geometry description of the parallel wire transmission line network. For the first scenario, (b) means and upper/lower variation bounds of $|V(\mathbf{x}, f)|$ and $|\tilde{V}(\mathbf{x}, f)|$, the PDFs of $|V(\mathbf{x}, f)|$ and $|\tilde{V}(\mathbf{x}, f)|$, at (c) $30.7692 \mathrm{MHz}$, (d) $60.7692 \mathrm{MHz}$, and (e) $90.7692 \mathrm{MHz}$, and (f) contributions of important random variables to the variance of $|\tilde{V}(\mathbf{x}, f)|$. For the second scenario, (g) PDFs of $|V(\mathbf{x}, f)|$ and $|\tilde{V}(\mathbf{x}, f)|$ at $20.2 \mathrm{MHz}$ and (h) contributions of important random variables to the variance of $|\tilde{V}(\mathbf{x}, f)|$ at $20.2 \mathrm{MHz}$. 
TABLE I

$\mu_{i}$ AND $\sigma_{i}, i=1, \ldots, 22$, VAlues of Normally Distributed RANDOM VARIables Considered in Both SCEnarios of Parallel Wire NETWORK EXAMPLE

\begin{tabular}{l|c|c} 
& $\mu_{i}$ & $\sigma_{i}$ \\
\hline$i=1, \ldots, 5, c x_{1}, c x_{2}, c x_{3}, c x_{4}, c x_{5}(\mathrm{~cm})$ & $(i-1) \times 3$ & 0.33 \\
\hline$i=6, \ldots, 10, c z_{1}, c z_{2}, c z_{3}, c z_{4}, c z_{5}(\mathrm{~cm})$ & 3 & 0.33 \\
\hline$i=11, \ldots, 15, R_{S, 1}, R_{S, 2}, R_{S, 3}, R_{S, 4}, R_{S, 5}(\Omega)$ & 50 & 3.3 \\
\hline$i=16, \ldots, 20, R_{L, 1}, R_{L, 2}, R_{L, 3}, R_{L, 4}, R_{L, 5}(\Omega)$ & 50 & 3.3 \\
\hline$i=21, L(\mu \mathrm{H})$ & 42.5 & 0.83 \\
\hline$i=22, C(\mathrm{pF})$ & 1.5 & 0.17
\end{tabular}

TABLE II

COMPARISON OF EFFICIENCY AND ACCURACY OF THE PROPOSED ME-PC ENHANCEd ITERATIVE HDMR METhod (COMBINED With PARTIAL FRACTION EXPANSION) AND OTHER METHODS IN CONSTRUCTING $\tilde{V}(\mathbf{x}, f)$ IN THE First SCENARIO OF THE PARALlEL Wire NETWORK EXAMPLE

\begin{tabular}{l|c|c}
\multicolumn{1}{c|}{ Method } & $\begin{array}{c}\text { Required number of } \\
V(\mathbf{x}, f) \text { samples }\end{array}$ & err \\
\hline $\begin{array}{l}\text { The proposed ME-PC enhanced iterative HDMR } \\
\text { technique (combined with partial fraction expansion) }\end{array}$ & 353 & $2.88 \times 10^{-3}$ \\
\hline Sparse grid based gPC collocation method & 921 & $2.12 \times 10^{-2}$ \\
\hline Sparse grid based ME-PC method & 697 & $1.66 \times 10^{-2}$ \\
\hline Adaptive sparse grid method & 395 & $2.82 \times 10^{-3}$ \\
\hline $\begin{array}{l}\text { The ME-PC enhanced iterative HDMR technique } \\
\text { (without partial fraction expansion) }\end{array}$ & 353 & $4.73 \times 10^{-3}$
\end{tabular}

first and fifth wires along the $x$-direction, $c x_{1}$ and $c x_{5}$, and the value of the resistor in Block $\mathrm{A}, R_{L, 5}$, have significant impact on $|V(\mathbf{x}, f)|$ at nonresonant frequencies. Almost all random variables and the second-order correlations of $c z_{1}, c z_{5}, c x_{1}$, and $c x_{5}$ significantly affect $|V(\mathbf{x}, f)|$ at resonant frequencies.

In addition, the efficiency and accuracy of the proposed ME-PC enhanced iterative HDMR technique (combined with partial fraction expansion) are compared with those of a sparse grid-based gPC collocation method utilizing second-order gPC expansion, a sparse grid-based ME-PC method utilizing first-order gPC expansions, the adaptive sparse grid method of [37] (with tolerance $10^{-1}$ ), and the ME-PC enhanced iterative HDMR technique (without partial fraction expansion). To this end, the surrogate model $\tilde{V}(\mathbf{x}, f)$ is constructed by each of these methods and used to obtain $\left|\tilde{V}\left(\mathbf{x}, f_{n}\right)\right|$, $n=1, \ldots, N_{f}, N_{f}=1001$, for $N_{\mathrm{MC}}=25000 \mathrm{MC}$ samples. The number of $V(\mathbf{x}, f)$ samples required to construct the surrogate model by each method and the average error in the surrogate model [computed using MC samples of $V\left(\mathbf{x}, f_{n}\right)$ and $\tilde{V}\left(\mathbf{x}, f_{n}\right)$ in (14)] are listed in Table II. Clearly, the proposed method requires half or fewer the number of $V(\mathbf{x}, f)$ samples that traditional gPC and ME-PC methods call for, while generating surrogate models that are more accurate by an order of magnitude. In addition, it requires slightly fewer samples than the adaptive sparse grid method for the same level of accuracy. The proposed ME-PC enhanced iterative HDMR technique (combined with partial fraction expansion) generates slightly more accurate surrogate models compared with the ME-PC enhanced iterative HDMR technique (without
TABLE III

COMPARISON OF EFFICIENCY AND ACCURACY OF THE PROPOSED ME-PC ENHANCED ITERATIVE HDMR METHOD AND NONADAPTIVE gPC COLLOCATION-BASED ITERATIVE HDMR METHOD IN COMPUTING

$|\tilde{V}(\mathbf{x}, f)|$ IN THE SECOND SCENARIO OF PARALLEL WIRE NETWORK EXAMPLE

\begin{tabular}{l|c|c}
\multicolumn{1}{c|}{ Method } & $\begin{array}{c}\text { Required number of } \\
|V(\mathbf{x}, f)| \text { samples }\end{array}$ & err \\
\hline ME-PC utilizing $2^{\text {nd }}$-order gPC expansions & 1539 & $6.27 \times 10^{-2}$ \\
\hline ME-PC utilizing $4^{\text {th }}$-order gPC expansions & 3115 & $3.22 \times 10^{-2}$ \\
\hline gPC collocation leveraging $4^{\text {th }}$-order gPC expansion & 1145 & 0.3681 \\
\hline gPC collocation leveraging 14 $4^{\text {th }}$-order gPC expansion & 13245 & $7.92 \times 10^{-2}$ \\
\hline gPC collocation leveraging $16^{\text {th }}$-order gPC expansion & 17249 & $6.14 \times 10^{-2}$ \\
\hline gPC collocation leveraging $19^{\text {th }}$-order gPC expansion & 26841 & $4.83 \times 10^{-2}$ \\
\hline gPC collocation leveraging $24^{\text {th }}$-order gPC expansion & 38545 & $3.71 \times 10^{-2}$
\end{tabular}

partial fraction expansion) as it uses vector fitting to interpolate over the frequency band. It should be mentioned that a straightforward tensor-product-based gPC collocation method utilizing second-order gPC expansion requires $3^{20}$ samples, and hence is not feasible for this high-dimensional problem (and for all other numerical examples in Section III). We note that the CPU time for surrogate model generation by all these nonintrusive methods (in all numerical examples in Section III) is nearly equal to the number of $V(\mathbf{x}, f)$ samples times, the CPU time required for the evaluation of one $V(\mathbf{x}, f)$ sample, which for this example is approximately 1.18 and $9.71 \mathrm{~s}$ for 75 and 1001 frequency points, respectively. Each $V(\mathbf{x}, f)$ sample is evaluated using the fast integral equation-based MTL simulator described in [41] after extracting the per-unit-length inductance and capacitance of the MTLs via a 2-D integral equation solver described in [53].

2) RLC Circuit Termination: In the second scenario, the fifth wire is terminated by Block B that consists of serially connected resistor $R_{L, 5}$, inductor $L$, and capacitor $C$ [Fig. 2(a)]. The voltage source is operated at the circuit's resonant frequency of $20.2 \mathrm{MHz}$. Twenty-two parameters characterize uncertainty in this network $\left(N_{\text {dof }}=22\right)$; those are $c x_{j}$ $(j=1, \ldots, 5), c z_{j}(j=1, \ldots, 5), R_{S, j}(j=1, \ldots, 5), R_{L, j}$ $(j=1, \ldots, 5)$, and the inductance and capacitance values, $L$ and $C ; \mathbf{x}=\left[c x_{1}, \ldots, c x_{5}, c z_{1}, \ldots, c z_{5}, R_{S, 1}, \ldots, R_{S, 5}\right.$, $\left.R_{L, 1}, \ldots, R_{L, 5}, L, C\right]$. All random variables are assumed normally distributed with means and standard deviations $\left(\mu_{i}, \sigma_{i}\right)(i=1, \ldots, 22)$ (Table I); each normal distribution again is approximated by a truncated Gaussian defined in range $\left[a_{i}, b_{i}\right]=\left[\mu_{i}-3 \sigma_{i}, \mu_{i}+3 \sigma_{i}\right]$ [51]. The observable is the crosstalk-induced voltage across the capacitor in Block B. The surrogate model $|\tilde{V}(\mathbf{x}, f)|$ at $20.2 \mathrm{MHz}$ is constructed using the proposed ME-PC enhanced iterative HDMR technique with $\varepsilon_{1}=10^{-3}$ and a nonadaptive gPC collocation-based iterative HDMR technique $\varepsilon_{1}=10^{-3}$. Table III shows the numbers of $|V(\mathbf{x}, f)|$ samples required by the ME-PC enhanced method utilizing second- and fourth-order gPC expansions, coefficients of which are obtained via tensor-product-based Gauss-Legendre quadrature rule with three and five points in each dimension, respectively. It also shows the numbers of $|V(\mathbf{x}, f)|$ samples required by $\mathrm{gPC}$ collocation-based method leveraging 4th-, 14th-, 
16th-, 19th-, and 24th-order gPC expansions, coefficients of which are obtained by tensor-product-based Gauss-Legendre quadrature rule with $5,15,17,20$, and 25 points in each dimension, respectively. In addition, $25000 \mathrm{MC}$ samples of $|V(\mathbf{x}, f)|$ and the surrogate model $|\tilde{V}(\mathbf{x}, f)|$ constructed by the ME-PC enhanced and $\mathrm{gPC}$ collocation-based methods are used in (14) $\left(N_{\mathrm{MC}}=25000\right)\left(N_{f}=1\right)$ and the error in the surrogate model constructed by these methods is tabulated in Table III. To construct the surrogate model $|\tilde{V}(\mathbf{x}, f)|$ with err $=0.06$ and err $=0.03$, the numbers of $|V(\mathbf{x}, f)|$ samples required by the proposed ME-PC enhanced iterative HDMR method are 1539 and 3115 while those required by the nonadaptive $\mathrm{gPC}$ collocation based iterative HDMR method are 17249 and 38545, respectively. Needless to say, the proposed ME-PC enhanced iterative HDMR method requires only a fraction of the $|V(\mathbf{x}, f)|$ samples required by the nonadaptive $\mathrm{gPC}$ collocation-based iterative HDMR method; the evaluation of one $|V(\mathbf{x}, f)|$ sample at $20.2 \mathrm{MHz}$ takes approximately $0.44 \mathrm{~s}$. Each $|V(\mathbf{x}, f)|$ sample is evaluated using the fast integral equation-based MTL simulator described in [41] after extracting the per-unit-length inductance and capacitance of the MTLs via a 2-D integral equation solver described in [53].

The PDFs of $|V(\mathbf{x}, f)|$ and the surrogate model $|\tilde{V}(\mathbf{x}, f)|$ constructed by the ME-PC enhanced iterative HDMR method (using five Gauss-Legendre points in each dimension) (at $20.2 \mathrm{MHz})$ are obtained from their MC samples [Fig. 2(g)]. The PDF of $|\tilde{V}(\mathbf{x}, f)|$ is nearly the same as that of $|V(\mathbf{x}, f)|$; this validates the accuracy of the proposed method. Moreover, global sensitivity indices of the random variables are computed via the surrogate model $|\tilde{V}(\mathbf{x}, f)|$ constructed by the ME-PC enhanced iterative HDMR method (utilizing five Gauss-Legendre points in each dimension) at 20.2 MHz [Fig. 2(h)] [52]. As the network is operated at the terminating circuit's resonance frequency, small perturbations in the capacitance value rapidly (and nonlinearly) change the crosstalk-induced voltage; this is why the capacitance value $C$ has the most significant impact on $|V(\mathbf{x}, f)|$. In addition to the capacitance value, the distances between the first, second, fourth, and fifth wires and the ground plane, $c z_{j}(j=1,2,4,5)$, and their second-order correlations with the capacitor value have significant impact on $|V(\mathbf{x}, f)|$.

\section{B. Parallel Interconnect Network}

The proposed method is next applied to the statistical characterization of crosstalk-induced voltage in a parallel interconnect network [Fig. 3(a)]. The network consists of 10 infinitesimally thin lossless 5 -cm-long conductors that reside on a lossless $100-\mu$ m-thick dielectric substrate with relative permittivity $\varepsilon_{r}=4.4$; the substrate is backed by an infinite PEC ground plane at $z=0 \mathrm{~m}$. The conductors have widths $w_{j}(j=1, \ldots, 10)$, are positioned parallel to each other, and are centered at $\left(c x_{j}, 0,100\right) \mu \mathrm{m}$ $(j=1, \ldots, 10)$. They are connected to the resistors at the source end, $R_{S, j}(j=1, \ldots, 10)$ and load end, $R_{L, j}(j=1, \ldots, 10)$ [Fig. 3(a)]. The first conductor in the network is excited by a sinusoidal voltage source operated in the $1-20-\mathrm{GHz}$ range.
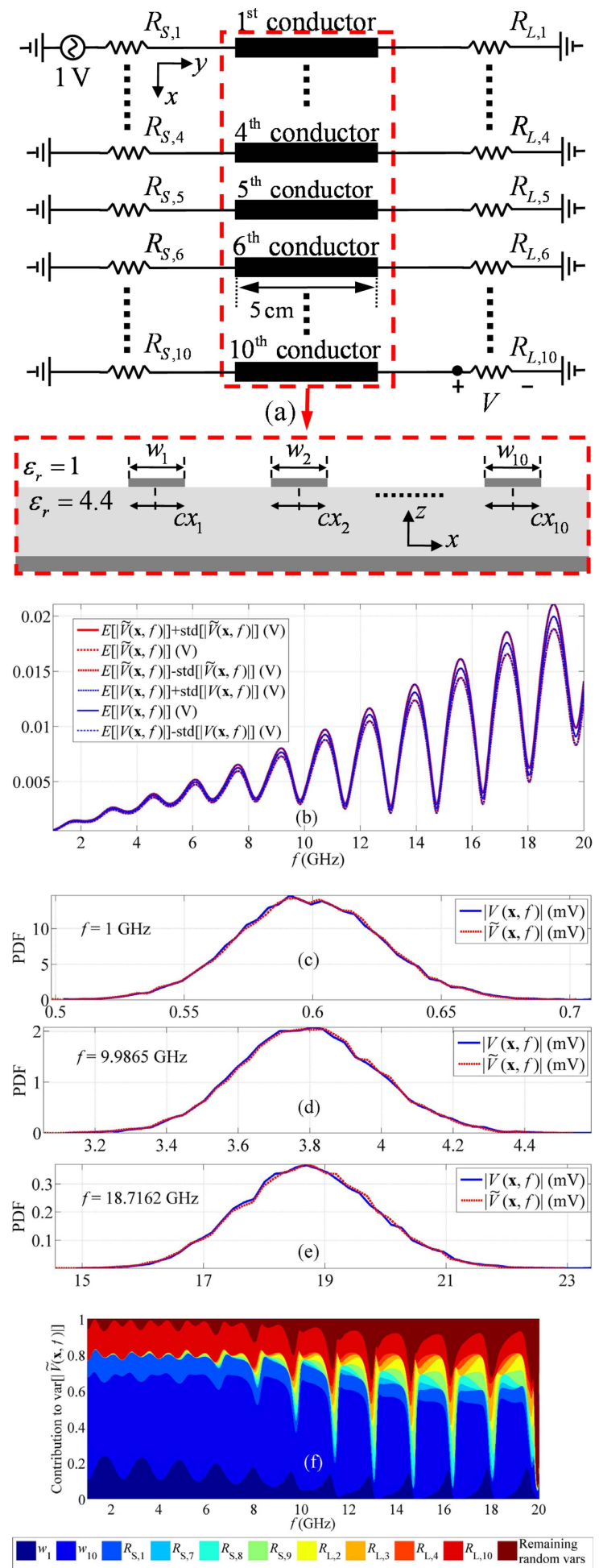

Fig. 3. (a) Geometry description of the parallel interconnect network. (b) Means and upper/lower variation bounds of $|V(\mathbf{x}, f)|$ and $|\tilde{V}(\mathbf{x}, f)|$, the crosstalk-induced voltage and its surrogate model. The PDFs of $|V(\mathbf{x}, f)|$ and $|\tilde{V}(\mathbf{x}, f)|$ at (c) $1 \mathrm{GHz}$, (d) $9.9865 \mathrm{GHz}$, and (e) $18.7162 \mathrm{GHz}$. (f) Contributions of important random variables to the variance of $|\tilde{V}(\mathbf{x}, f)|$.

Forty parameters characterize uncertainty in the network $\left(N_{\mathrm{dof}}=40\right)$. They are the positions of the conductors along the $x$-direction $c x_{j}(j=1, \ldots, 10)$, the widths of the conductors $w_{j}(j=1, \ldots, 10)$, the values of the resistors at the source end $R_{S, j}(j=1, \ldots, 10)$, and the values of the resistors at the 
TABLE IV

$\mu_{i}$ AND $\sigma_{i}, i=1, \ldots, 40$, VAlues of Normally Distributed Random VARIABLES IN PARALLEL INTERCONNECT NETWORK EXAMPLE

\begin{tabular}{l|c|c} 
& $\mu_{i}$ & $\sigma_{i}$ \\
\hline$i=1, \ldots, 10, d x_{1}, d x_{2}, d x_{3}, d x_{4}, d x_{5}, d x_{6}, d x_{7}, d x_{8}, d x_{9}, d x_{10}(\mu \mathrm{m})$ & $(i-1) \times 234.1$ & 3.3 \\
\hline$i=11, \ldots, 20, w_{1}, w_{2}, w_{3}, w_{4}, w_{5}, w_{6}, w_{7}, w_{8}, w_{9}, w_{10}(\mu \mathrm{m})$ & 134.1 & 3.3 \\
\hline$i=21, \ldots, 30, R_{S, 1}, R_{S, 2}, R_{S, 3}, R_{S, 4}, R_{S, 5}, R_{S, 6}, R_{S, 7}, R_{S, 8}, R_{S, 9}, R_{S, 10}(\Omega)$ & 50 & 3.3 \\
\hline$i=31, \ldots, 40, R_{L, 1}, R_{L, 2}, R_{L, 3}, R_{L, 4}, R_{L, 5}, R_{L, 6}, R_{L, 7}, R_{L, 8}, R_{L, 9}, R_{L, 10}(\Omega)$ & 50 & 3.3
\end{tabular}

TABLE V

COMPARISON OF EFFICIENCY AND ACCURACY OF THE PROPOSED ME-PC ENHANCED ITERATIVE HDMR METHOd (COMBINED With PARTIAL FraCtion EXPANSION) AND OTHER METHODS IN CONSTRUCTING $\tilde{V}(\mathbf{x}, f)$ in the Parallel Interconnect Network Example

\begin{tabular}{l|c|c}
\multicolumn{1}{c|}{ Method } & $\begin{array}{c}\text { Required number of } \\
V(\mathbf{x}, f) \text { samples }\end{array}$ & err \\
\hline $\begin{array}{l}\text { The proposed ME-PC enhanced iterative HDMR } \\
\text { technique (combined with partial fraction expansion) }\end{array}$ & 81 & $1.14 \times 10^{-3}$ \\
\hline Sparse grid based gPC collocation method & 3441 & $8.49 \times 10^{-3}$ \\
\hline Adaptive sparse grid method & 81 & $2.55 \times 10^{-3}$ \\
\hline $\begin{array}{l}\text { The ME-PC enhanced iterative HDMR technique } \\
\text { (without partial fraction expansion) }\end{array}$ & 81 & $2.31 \times 10^{-3}$
\end{tabular}

load end $R_{L, j}(j=1, \ldots, 10) ;\left(\mathbf{x}=\left[c x_{1}, \ldots, c x_{10}, w_{1}, \ldots\right.\right.$, $\left.\left.w_{10}, R_{S, 1}, \ldots, R_{S, 10}, R_{L, 1}, \ldots, R_{L, 10}\right]\right)$. All random variables are assumed normally distributed with means and standard deviations $\left(\mu_{i}, \sigma_{i}\right)(i=1, \ldots, 40)$ specified in Table IV. It should be noted here that the random variables' means correspond to those in [1] and each normal distribution is approximated by a truncated Gaussian defined in the range $\left[a_{i}, b_{i}\right]=\left[\mu_{i}-3 \sigma_{i}, \mu_{i}+3 \sigma_{i}\right]$ [51]. The observable is the crosstalk-induced voltage across the resistor at the load end of the tenth conductor. The ME-PC enhanced iterative HDMR technique (combined with partial fraction expansion) required 81 samples of $V(\mathbf{x}, f)$ at 75 frequency points $\left(N_{s}=75\right)$ to construct the surrogate model $\tilde{V}(\mathbf{x}, f)$; the adaptive ME-PC used second-order gPC expansions, coefficients of which are obtained via tensor-product-based Gauss-Legendre quadrature rule with three points in each dimension.

The means and variation bounds of $\left|V\left(\mathbf{x}, f_{n}\right)\right|$ and $\left|\tilde{V}\left(\mathbf{x}, f_{n}\right)\right|, n=1, \ldots, N_{f}, N_{f}=1001$, which are computed using an MC method with $N_{\mathrm{MC}}=25000$ samples, are identical over the entire frequency band [Fig. 3(b)]. MC samples of $V\left(\mathbf{x}, f_{n}\right)$ and $\tilde{V}\left(\mathbf{x}, f_{n}\right)$ are then used in (14) and the error is found to be err $=1.14 \times 10^{-3}$, verifying the accuracy of the proposed techniques.

The PDFs of $|V(\mathbf{x}, f)|$ and $|\tilde{V}(\mathbf{x}, f)|$ at three frequency points are extracted by binning the samples obtained in the MC simulations [Fig. 3(c)-(e)]. The PDFs of $|\tilde{V}(\mathbf{x}, f)|$ match those of $|V(\mathbf{x}, f)|$. Furthermore, the contributions of random variables to the variance of $|V(\mathbf{x}, f)|$ are computed using the surrogate models [Fig. 3(f)]. Apparently, contributions of uncertainties in the widths of the first and tenth conductors $\left(w_{1}\right.$ and $\left.w_{10}\right)$, the value of the resistor at the source end of first conductor $R_{S, 1}$, and the value of the resistor at the load end of tenth conductor $R_{L, 10}$, dominate observer variability at lower frequencies. As the frequency increases, other random variables (specifically, $R_{S, 7}, R_{S, 8}, R_{S, 9}, R_{L, 2}, R_{L, 3}$, and $R_{L, 4}$ ) exhibit significant impact on $|V(\mathbf{x}, f)|$ as well.
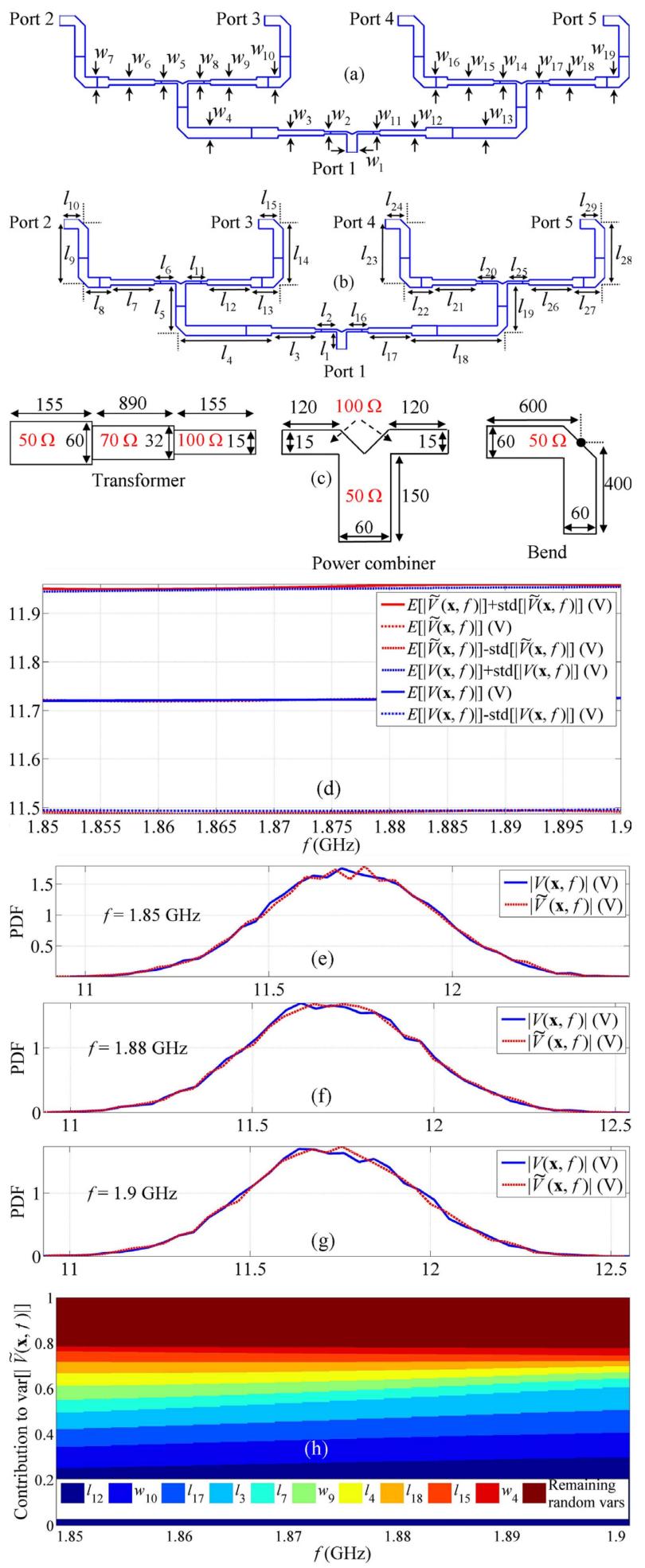

Fig. 4. Geometry description of the corporate feed network with indicated interconnect (a) widths and (b) lengths. (c) Geometry description and intrinsic impedances of the transformer, power combiner, and bend that are used to construct corporate feed network (all dimensions are in mils). (d) Means and upper/lower variation bounds of $|V(\mathbf{x}, f)|$ and $|\tilde{V}(\mathbf{x}, f)|$, the crosstalkinduced voltage and its surrogate model. The PDFs of $|V(\mathbf{x}, f)|$ and $|\tilde{V}(\mathbf{x}, f)|$ at (e) $1.85 \mathrm{GHz}$, (f) $1.88 \mathrm{GHz}$, and (g) $1.9 \mathrm{GHz}$. (h) Contributions of important random variables to the variance of $|\tilde{V}(\mathbf{x}, f)|$.

Furthermore, the efficiency and accuracy of the proposed ME-PC enhanced iterative HDMR technique (combined with partial fraction expansion) are compared with those 
This article has been accepted for inclusion in a future issue of this journal. Content is final as presented, with the exception of pagination.

TABLE VI

$a_{i}$ AND $b_{i}, i=1, \ldots, 48$, Values of Uniformly Distributed Random Variables in Corporate Feed Network Example (IN Mils)

\begin{tabular}{|c|c|c|c|c|c|c|c|c|c|c|c|}
\hline & $a_{i}$ & $b_{i}$ & & $a_{i}$ & $b_{i}$ & & $a_{i}$ & $b_{i}$ & & $a_{i}$ & $b_{i}$ \\
\hline$i=1, w_{1}$ & 57 & 63 & $i=13, w_{13}$ & 57 & 63 & $i=25, l_{6}$ & 261.25 & 288.75 & $i=37, l_{18}$ & 2189.75 & 2420.25 \\
\hline$i=2, w_{2}$ & 14.25 & 15.75 & $i=14, w_{14}$ & 14.25 & 15.75 & $i=26, l_{7}$ & 845.5 & 934.5 & $i=38, l_{19}$ & 712.5 & 787.5 \\
\hline$i=4, w_{4}$ & 57 & 63 & $i=16, w_{16}$ & 57 & 63 & $i=28, l_{9}$ & 1140 & 1260 & $i=40, l_{21}$ & 845.5 & 934.5 \\
\hline$i=5, w_{5}$ & 14.25 & 15.75 & $i=17, w_{17}$ & 14.25 & 15.75 & $i=29, l_{10}$ & 380 & 420 & $i=41, l_{22}$ & 527.25 & 582.75 \\
\hline$i=8, w_{8}$ & 14.25 & 15.75 & $i=20, l_{1}$ & 142.5 & 157.5 & $i=32, l_{13}$ & 527.25 & 582.75 & $i=44, l_{25}$ & 261.25 & 288.75 \\
\hline$i=9, w_{9}$ & 30.4 & 33.6 & $i=21, l_{2}$ & 261.25 & 288.75 & $i=33, l_{14}$ & 1140 & 1260 & $i=45, l_{26}$ & 845.5 & 934.5 \\
\hline$i=10, w_{10}$ & 57 & 63 & $i=22, l_{3}$ & 845.5 & 934.5 & $i=34, l_{15}$ & 380 & 420 & $i=46, l_{27}$ & 527.25 & 582.75 \\
\hline$i=11, w_{11}$ & 14.25 & 15.75 & $i=23, l_{4}$ & 2189.75 & 2420.25 & $i=35, l_{16}$ & 261.25 & 288.75 & $i=47, l_{28}$ & 1140 & 1260 \\
\hline
\end{tabular}

of a sparse grid-based gPC collocation method utilizing second-order gPC expansion, adaptive sparse grid method of [37] (with tolerance $10^{-1}$ ), and the ME-PC enhanced iterative HDMR technique (without partial fraction expansion). To this end, each method is used to construct the surrogate model $\tilde{V}(\mathbf{x}, f)$, which is then probed to obtain $\left|\tilde{V}\left(\mathbf{x}, f_{n}\right)\right|$, $n=1, \ldots, N_{f}, N_{f}=1001$, for $N_{\mathrm{MC}}=25000 \mathrm{MC}$ samples. The number of $V(\mathbf{x}, f)$ samples required to construct the surrogate model by each method and the average error in the surrogate model [computed using MC samples of $V\left(\mathbf{x}, f_{n}\right)$ and $\tilde{V}\left(\mathbf{x}, f_{n}\right)$ in (14)] are listed in Table V. Apparently, all methods produce surrogate models with roughly the same level of accuracy, with the proposed method producing the most accurate one. The sparse grid-based gPC collocation method requires 42.5 times more $V(\mathbf{x}, f)$ samples compared with the proposed method and the adaptive sparse grid method. Note that the evaluation of one $V(\mathbf{x}, f)$ sample at 75 and 1001 frequency points requires approximately 7.85 and $26.25 \mathrm{~s}$, respectively. Each $V(\mathbf{x}, f)$ sample is evaluated using the fast integral equation-based MTL simulator described in [41] after extracting the per-unit-length inductance and capacitance of the MTLs via a 2-D integral equation solver described in [53].

\section{Corporate Feed Network}

Finally, the proposed method is used to compute statistics of a port voltage in a corporate feed network of a linear patch antenna array [Fig. 4(a) and (b)]. The network feeds four patch antennas at $1.88 \mathrm{GHz}$ [54] and is constructed by cascading a transformer, a power combiner, and a bend [Fig. 4(c)]. These components reside on a lossless 31-mils-thick dielectric substrate with relative permittivity $\varepsilon_{r}=4.4$ that is backed by an infinite PEC ground plane at $z=0$ mils [54]. All ports of the network are terminated by $50-\Omega$ resistors and the network is excited at the first port by a 1-A sinusoidal current source operated in the 1.85-1.9-GHz range.

Forty-eight parameters characterize the uncertainty in the corporate feed network $\left(N_{\text {dof }}=48\right)$. They are the widths of all conductors $\left(w_{j}, j=1, \ldots, 19\right)$ and their lengths $\left(l_{j}, j=1, \ldots, 29\right) ;\left(\mathbf{x}=\left[w_{1}, \ldots, w_{19}, l_{1}, \ldots, l_{29}\right]\right)$ [Fig. 4(a) and (b)]. All random variables are assumed uniformly distributed in the ranges $\left[a_{i}, b_{i}\right], i=1, \ldots, 48$ specified in Table VI. It should be noted here that the random variables' means correspond to widths and lengths of the corporate feed network in [54] and $a_{i}$ and $b_{i}$ are obtained by adding $\mp \% 5$ variation to each random variable's mean. The observable is the voltage across the second port of the network. The ME-PC enhanced iterative HDMR technique used second-order gPC expansions, coefficients of which are obtained via tensor-product-based Gauss-Legendre quadrature with three points in each dimension, and required 97 samples of $V(\mathbf{x}, f)$ to construct the surrogate model $\tilde{V}(\mathbf{x}, f)$; the evaluation of one $V(\mathbf{x}, f)$ sample takes approximately $9.46 \mathrm{~s}$. Each $V(\mathbf{x}, f)$ sample is evaluated using IE3D full-wave solver [42].

The means and variation bounds of $\left|V\left(\mathbf{x}, f_{n}\right)\right|$ and $\left|\tilde{V}\left(\mathbf{x}, f_{n}\right)\right|, n=1, \ldots, N_{f}, N_{f}=21$, are computed using an MC method with $N_{\mathrm{MC}}=25000$ samples [Fig. 4(d)]. Obviously, statistics of $\left|V\left(\mathbf{x}, f_{n}\right)\right|$ and $\left|\tilde{V}\left(\mathbf{x}, f_{n}\right)\right|$ match well. The MC samples are used in (14) and the error is found to be err $=5.41 \times 10^{-3}$. The PDFs of $|V(\mathbf{x}, f)|$ and $|\tilde{V}(\mathbf{x}, f)|$ at three frequencies are extracted by binning the samples obtained in the MC simulations [Fig. 4(e)-(g)]. The PDFs of $|\tilde{V}(\mathbf{x}, f)|$ match those of $|V(\mathbf{x}, f)|$. Furthermore, contributions of the random variables to the variance of $|V(\mathbf{x}, f)|$ are computed using (11) [Fig. 4(h)]. The random variables describing the widths $w_{4}, w_{9}$, and $w_{10}$, the lengths of the transformers $l_{3}, l_{7}, l_{12}$, and $l_{17}$, and the lengths of the bend sections $l_{4}, l_{15}$, and $l_{18}$ have significant impact on $|V(\mathbf{x}, f)|$; the impact of these random variables is prominent throughout the frequency band.

\section{CONCLUSion}

A computational framework leveraging HDMR expansions is proposed to statistically characterize MTL networks subject to a large number of manufacturing uncertainties. The framework builds surrogate models of observables in 
MTL networks by iteratively constructing HDMR component functions pertinent to the most significant random variables. Each component function of the HDMR expansions is approximated by the ME-PC method. The observable values at integration/collocation points identified by the ME-PC method are computed via an integral equation MTL simulator. The computational framework combines the HDMR and partial fraction expansions for broadband stochastic MTL analysis. When compared with traditional gPC Galerkin and collocation methods including their $h$-adaptive extensions, the proposed computational framework is both efficient and accurate when generating surrogate models of smoothly and rapidly varying observables in high-dimensional random domains. The proposed methodology was applied to stochastic MTL scenarios involving 20-50 random variables but remains useful in scenarios involving hundreds of random variables [55], [56].

\section{REFERENCES}

[1] E. Laermans, F. Olyslager, and D. De Zutter, "Sensitivity based statistical analysis of multiconductor transmission lines in multilayered media," in Proc. Int. Symp. AP-S, Jul. 1996, pp. 288-291.

[2] D. Bellan, S. A. Pignari, and G. Spadacini, "Characterisation of crosstalk in terms of mean value and standard deviation [multiconductor transmission lines]," IEE Proc.-Sci. Meas. Technol., vol. 150, no. 6, pp. 289-295, Nov. 2003.

[3] A. Ciccolella and F. G. Canavero, "Stochastic prediction of wire coupling interference," in Proc. IEEE Int. Symp. EMC, Aug. 1995, pp. 51-56.

[4] T. Mikazuki and N. Matsui, "Statistical design techniques for highspeed circuit boards with correlated structure distributions," IEEE Trans. Compon., Packag., Manuf. Technol. A, vol. 17, no. 1, pp. 159-165, Mar. 1994.

[5] G. Fishman, Monte Carlo: Concepts, Algorithms, and Applications. Berlin, Germany: Springer-Verlag, 1995.

[6] H. Bagci, A. C. Yucel, J. S. Hesthaven, and E. Michielssen, "A fast stroud-based collocation method for statistically characterizing EMI/EMC phenomena on complex platforms," IEEE Trans. Electromagn. Compat., vol. 51, no. 2, pp. 301-311, May 2009.

[7] A. C. M. Austin and C. D. Sarris, "Efficient analysis of geometrical uncertainty in the FDTD method using polynomial chaos with application to microwave circuits," IEEE Trans. Microw. Theory Techn., vol. 61, no. 12, pp. 4293-4301, Dec. 2013.

[8] H. Bagci, C. Yavuz, A. C. Yucel, J. S. Hesthaven, and E. Michielssen, "A fast and parallel stroud-based stochastic collocation method for statistical EMI/EMC analysis," in Proc. IEEE Int. Symp. EMC, Detroit, MI, USA, Aug. 2008, pp. 1-5.

[9] A. C. M. Austin, N. Sood, J. Siu, and C. D. Sarris, "Application of polynomial chaos to quantify uncertainty in deterministic channel models," IEEE Trans. Antennas Propag., vol. 61, no. 11, pp. 5754-5761, Nov. 2013.

[10] A. C. Yucel, Y. Liu, H. Bagci, and E. Michielssen, "Statistical characterization of electromagnetic wave propagation in mine environments," IEEE Antennas Wireless Propag. Lett., vol. 12, pp. 1602-1605, Dec. 2013.

[11] F. Boeykens, H. Rogier, and L. Vallozzi, "An efficient technique based on polynomial chaos to model the uncertainty in the resonance frequency of textile antennas due to bending," IEEE Trans. Antennas Propag., vol. 62, no. 3, pp. 1253-1260, Mar. 2014

[12] O. Bakir, A. C. Yucel, E. Michielssen, and H. Bagci, "Statistical characterization of wave propagation in mine environments," in Proc. Conf. Held, Chicago, IL, USA, Jul. 2012, pp. 1-2.

[13] L. J. Gomez, A. C. Yucel, L. Hernandez-Garcia, S. F. Taylor, and E. Michielssen, "Uncertainty quantification in transcranial magnetic stimulation via high-dimensional model representation," IEEE Trans. Biomed. Eng., vol. 62, no. 1, pp. 361-372, Jan. 2015.

[14] L. R. A. X. de Menezes, A. Ajayi, C. Christopoulos, P. Sewell, and G. A. Borges, "Efficient computation of stochastic electromagnetic problems using unscented transforms," IET Sci., Meas. Technol., vol. 2, no. 2, pp. 88-95, Mar. 2008.
[15] L. J. Gomez, A. C. Yucel, L. Hernandez-Garcia, S. F. Taylor, and E. Michielssen, "Sensitivity of TMS-induced electric fields to the uncertainty in coil placement and brain anatomy," in Proc. IEEE AP-S. Int. Symp., Jul. 2014, pp. 5-6.

[16] A. C. Yucel, H. Bagci, and E. Michielssen, "Fast probability density function estimation for statistical EMC/EMI characterization," presented at the USNC/URSI Nat. Radio Sci. Meeting, Charleston, SC, USA, 2009.

[17] D. Xiu, "Fast numerical methods for stochastic computations: A review," Commun. Comput. Phys., vol. 5, nos. 2-4, pp. 242-272, 2009.

[18] I. S. Stievano, P. Manfredi, and F. G. Canavero, "Stochastic analysis of multiconductor cables and interconnects," IEEE Trans. Electromagn. Compat., vol. 53, no. 2, pp. 501-507, May 2011.

[19] I. S. Stievano, P. Manfredi, and F. G. Canavero, "Parameters variability effects on multiconductor interconnects via Hermite polynomial chaos," IEEE Trans. Compon., Packag., Manuf. Technol., vol. 1, no. 8, pp. 1234-1239, Aug. 2011.

[20] D. Vande Ginste, D. De Zutter, D. Deschrijver, T. Dhaene, P. Manfredi, and F. Canavero, "Stochastic modeling-based variability analysis of onchip interconnects," IEEE Trans. Compon., Packag., Manuf. Technol., vol. 2, no. 7, pp. 1182-1192, Jul. 2012.

[21] A. Biondi, D. Vande Ginste, D. De Zutter, P. Manfredi, and F. G. Canavero, "Variability analysis of interconnects terminated by general nonlinear loads," IEEE Trans. Compon., Packag., Manuf. Technol., vol. 3, no. 7, pp. 1244-1251, Jul. 2013.

[22] T.-A. Pham, E. Gad, M. S. Nakhla, and R. Achar, "Decoupled polynomial chaos and its applications to statistical analysis of high-speed interconnects," IEEE Trans. Compon., Packag., Manuf. Technol., vol. 4, no. 10 , pp. 1634-1647, Oct. 2014.

[23] P. Manfredi, D. Vande Ginste, D. De Zutter, and F. G. Canavero, "Stochastic modeling of nonlinear circuits via SPICE-compatible spectral equivalents," IEEE Trans. Circuits Syst. I, Reg. Papers, vol. 61, no. 7, pp. 2057-2065, Jul. 2014.

[24] F. Diouf and F. Canavero, "Crosstalk statistics via collocation method," in Proc. IEEE Int. Symp. EMC, Austin, TX, USA, Aug. 2009, pp. 92-97.

[25] A. Rong and A. C. Cangellaris, "Interconnect transient simulation in the presence of layout and routing uncertainty," in Proc. IEEE 20th Conf. Elect. Perform. Electron. Packag. Syst. (EPEPS), Oct. 2011, pp. $157-160$.

[26] C. J. Hyung and A. C. Cangellaris, "Fast assessment of the impact of surrounding wiring on the transmission properties of high-speed interconnect channels," in Proc. IEEE 21st Conf. Elect. Perform. Electron. Packag. Syst. (EPEPS), Oct. 2012, pp. 69-72.

[27] D. Spina, F. Ferranti, G. Antonini, T. Dhaene, and L. Knockaert, "Non intrusive polynomial chaos-based stochastic macromodeling of multiport systems," in Proc. IEEE 18th Workshop Signal Power Integr. (SPI), May 2014, pp. 1-4.

[28] M. Kabir et al., "Non-intrusive pseudo spectral approach for stochastic macromodeling of EM systems using deterministic full-wave solvers," in Proc. 23rd Conf. EPEPS, 2014, pp. 1-4.

[29] X. Wan and G. E. Karniadakis, "An adaptive multi-element generalized polynomial chaos method for stochastic differential equations," J. Comput. Phys., vol. 209, no. 2, pp. 617-642, 2005.

[30] J. Foo, X. Wan, and G. E. Karniadakis, "The multi-element probabilistic collocation method (ME-PCM): Error analysis and applications," J. Comput. Phys., vol. 227, no. 22, pp. 9572-9595, 2008.

[31] J. Foo and G. E. Karniadakis, "Multi-element probabilistic collocation method in high dimensions," J. Comput. Phys., vol. 229, no. 5, pp. 1536-1557, 2010.

[32] X. Ma and N. Zabaras, "An adaptive high-dimensional stochastic model representation technique for the solution of stochastic partial differential equations," J. Comput. Phys., vol. 229, no. 10, pp. 3884-3915, 2010.

[33] J. De Geest, T. Dhaene, N. Faché, and D. De Zutter, "Adaptive CAD-model building algorithm for general planar microwave structures," IEEE Trans. Microw. Theory Techn., vol. 47, no. 9, pp. 1801-1809, Sep. 1999.

[34] R. Achar and M. S. Nakhla, "Simulation of high-speed interconnects," Proc. IEEE, vol. 89, no. 5, pp. 693-728, May 2001.

[35] T. El-Moselhy and L. Daniel, "Variation-aware interconnect extraction using statistical moment preserving model order reduction," in Proc. IEEE Conf. Design, Autom. Test Eur. Conf. Exhibit. (DATE), Mar. 2010, pp. $453-458$.

[36] Z. Zhang, T. A. El-Moselhy, I. M. Elfadel, and L. Daniel, "Stochastic testing method for transistor-level uncertainty quantification based on generalized polynomial chaos," IEEE Trans. Comput.-Aided Design Integr. Circuits Syst., vol. 32, no. 10, pp. 1533-1545, Oct. 2013. 
[37] J. S. Ochoa and A. C. Cangellaris, "Random-space dimensionality reduction for expedient yield estimation of passive microwave structures," IEEE Trans. Microw. Theory Techn., vol. 61, no. 12, pp. 4313-4321, Dec. 2013.

[38] H. Rabitz, Ö. F. Alis, J. Shorter, and K. Shim, "Efficient input-output model representations," Comput. Phys. Commun., vol. 117, nos. 1-2, pp. 11-20, 1999.

[39] A. C. Yucel, H. Bagci, and E. Michielssen, "An $h$-adaptive stochastic collocation method for stochastic EMC/EMI analysis," in Proc. IEEE Int. Symp. Antennas Propag., Jul. 2010, pp. 1-4.

[40] A. C. Yucel, H. Bagci, and E. Michielssen, "An adaptive multi-element probabilistic collocation method for statistical EMC/EMI characterization," IEEE Trans. Electromagn. Compat., vol. 55, no. 6, pp. 1154-1168, Dec. 2013.

[41] H. Bagci, A. E. Yilmaz, and E. Michielssen, "An FFT-accelerated time-domain multiconductor transmission line simulator," IEEE Trans. Electromagn. Compat., vol. 52, no. 1, pp. 199-214, Feb. 2010.

[42] IE3D Software Release 15.3, Mentor Graphics Corp., Wilsonville, OR, USA, 2014.

[43] B. Gustavsen and A. Semlyen, "Rational approximation of frequency domain responses by vector fitting," IEEE Trans. Power Del., vol. 14, no. 3, pp. 1052-1061, Jul. 1999.

[44] B. Gustavsen, "Improving the pole relocating properties of vector fitting," IEEE Trans. Power Del., vol. 21, no. 3, pp. 1587-1592, Jul. 2006.

[45] D. Deschrijver, M. Mrozowski, T. Dhaene, and D. De Zutter, "Macromodeling of multiport systems using a fast implementation of the vector fitting method," IEEE Microw. Wireless Compon. Lett., vol. 18, no. 6, pp. 383-385, Jun. 2008.

[46] I. M. Sobol, "Theorems and examples on high dimensional model representation," Rel. Eng. Syst. Safety, vol. 79, no. 2, pp. 187-193, 2003.

[47] V. I. Krylov, Approximate Calculation of Integrals. New York, NY, USA: Macmillan, 1962.

[48] T. Gerstner and M. Griebel, "Dimension-adaptive tensor-product quadrature," Computing, vol. 71, no. 1, pp. 65-87, 2003.

[49] T. Gerstner and M. Griebel, "Numerical integration using sparse grids," Numer. Algorithms, vol. 18, nos. 3-4, pp. 209-232, 1998.

[50] The Vector Fitting Web Site. [Online]. Available: https://www.sintef.no/projectweb/vectfit/, accessed Apr. 28, 2015.

[51] N. L. Johnson and S. Kotz, Continuous Univariate Distributions, vol. 1. New York, NY, USA: Wiley, 1970.

[52] T. Homma and A. Saltelli, "Importance measures in global sensitivity analysis of nonlinear models," Rel. Eng. Syst. Safety, vol. 52, no. 1, pp. 1-17, 1996.

[53] C. R. Paul, Analysis of Multiconductor Transmission Lines. Hoboken, NJ, USA: Wiley, 2008.

[54] IE3D User's Manual Release 14.1, Zeland Software, Inc., Fremont, CA, USA, May 2008.

[55] A. C. Yucel, H. Bagci, and E. Michielssen, "Stochastic electromagnetic analysis via high dimensional model representations," presented at the CNC-USNC/URSI Nat. Radio Sci. Meeting, Toronto, ON, Canada, 2010.

[56] A. C. Yucel, H. Bagci, and E. Michielssen, "Efficient stochastic EMC/EMI analysis using HDMR-generated surrogate models," in Proc. 30th URSI General Assembly Sci. Symp., Istanbul, Turkey, Aug. 2011, pp. $1-4$.

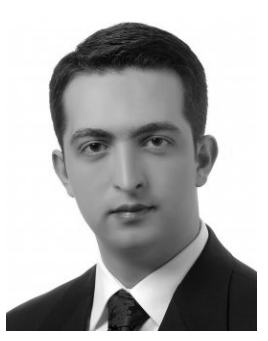

Abdulkadir C. Yücel received the B.S. (summa cum laude) degree in electronics engineering from the Gebze Institute of Technology, Kocaeli, Turkey, in 2005, and the M.S. and Ph.D. degrees in electrical engineering from the University of Michigan, Ann Arbor, MI, USA, in 2008 and 2013, respectively.

He was a Research and Teaching Assistant with the Department of Electronics Engineering, Gebze Institute of Technology, from 2005 to 2006. From 2006 to 2013, he was a Graduate Student Research Assistant with the Radiation Laboratory, University of Michigan, where he has been a Research Fellow since 2013. His current research interests include various aspects of computational electromagnetics with an emphasis on uncertainty quantification for electromagnetic analysis on complex platforms, electromagnetic compatibility and interference analysis, nature-based design of electromagnetic devices, and integral equation-based frequency and time domain solvers and their accelerators.

Dr. Yücel received the Fulbright Fellowship in 2006, and the Electrical Engineering and Computer Science Departmental Fellowship of the University of Michigan in 2007.

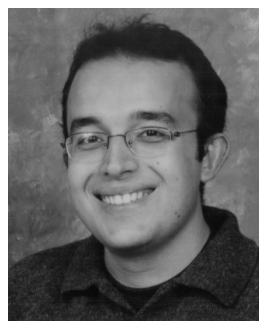

Hakan Bağcı (SM'14) received the B.S. degree in electrical and electronics engineering from Bilkent University, Ankara, Turkey, in 2001, and the M.S. and Ph.D. degrees in electrical and computer engineering from the University of Illinois at Urbana-Champaign (UIUC), Champaign, IL, USA, in 2003 and 2007, respectively.

$\mathrm{He}$ was an Undergraduate Researcher with the Computational Electromagnetics Group, Bilkent University, from 1999 to 2001. From 2001 to 2006, he was a Research Assistant with the Center for Computational Electromagnetics and the Electromagnetics Laboratory, UIUC. From 2007 to 2009, he was a Research Fellow with the Radiation Laboratory, University of Michigan, Ann Arbor, MI, USA. In 2009, he joined the Division of Physical Sciences and Engineering, King Abdullah University of Science and Technology, Jeddah, Saudi Arabia, as an Assistant Professor of Electrical Engineering. His current research interests include various aspects of computational electromagnetics with an emphasis on time domain integral equations and their fast marching-on-in-time-based solutions, well-conditioned integral-equation formulations, and development of fast hybrid methods for analyzing statistical electromagnetic compatibility (EMC)/electromagnetic interference (EMI) phenomena on complex and fully loaded platforms.

Dr. Bağcı was a recipient of the International Union of Radio Scientists Young Scientist Award in 2008, and the Interdisciplinary Graduate Fellowship from the Department of Computational Science and Engineering, UIUC, from 2004 to 2005. His paper titled Fast and rigorous analysis of EMC/EMI phenomena on electrically large and complex structures loaded with coaxial cables was one of the three finalists (with honorable mention) for the Richard B. Schulz Best Transactions Paper Award by the IEEE Electromagnetic Compatibility Society in 2008. He authored and co-authored nine finalist papers in the student paper competitions at the IEEE Antennas and Propagation Society International Symposiums in 2005, 2008, 2010, and 2014, and the Applied Computational Electromagnetics Society Conferences in 2013 and 2014.

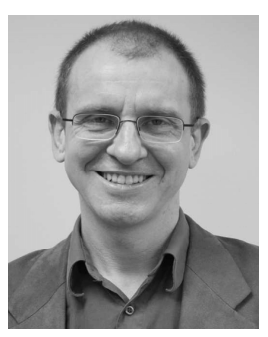

Eric Michielssen (F'02) received the M.S. (summa cum laude) degree in electrical engineering from the Katholieke Universiteit Leuven, Leuven, Belgium, in 1987, and the Ph.D. degree in electrical engineering from the University of Illinois at UrbanaChampaign (UIUC), Champaign, IL, USA, in 1992.

He joined as a Faculty Member with the Department of Electrical and Computer Engineering, UIUC, in 1993, where he held the rank of Full Professor in 2002. In 2005, he joined the University of Michigan (UM), Ann Arbor, MI, USA, as a Professor of Electrical Engineering and Computer Science. Since 2013, he has served as the UM's Associate Vice President for Advanced Research Computing. He currently directs the Michigan Institute for Computational Discovery and Engineering. He has authored or co-authored over 170 journal papers and book chapters, and over 300 papers in conference proceedings. His research focuses on the development of fast frequency and time domain integral-equation-based techniques for analyzing electromagnetic phenomena, and the development of robust optimizers for the synthesis of electromagnetic/ optical devices. His current research interests include all aspects of theoretical and applied computational electromagnetics.

Dr. Michielssen is a member of the URSI Commission B. He received the Belgian American Educational Foundation Fellowship in 1988, and the Schlumberger Fellowship in 1990. He was a recipient of the International Union of Radio Scientists (URSI) Young Scientist Fellowship in 1994, the National Science Foundation CAREER Award in 1995, and the Applied Computational Electromagnetics Society (ACES) Valued Service Award in 1998. In addition, he was named 1999 URSI United States National Committee Henry G. Booker Fellow, and selected as a recipient of the 1999 URSI Koga Gold Medal. He was also a recipient of the UIUC's Xerox Award for Faculty Research in 2001, the UM College of Engineering David E. Liddle Research Excellence Award in 2011, and the IEEE Antenna and Propagation Society Chen To Tai Distinguished Educator Award in 2014. He was appointed as a Beckman Fellow with the Center for Advanced Studies, UIUC, in 2002. He was named 2003 Scholar with the Tel Aviv University Sackler Center for Advanced Studies, and selected as a UIUC 2003 University and Sony Scholar. $\mathrm{He}$ served as the Technical Chairman of the ACES Symposium (Review of Progress in Applied Computational Electromagnetics in 1997, Monterey, CA, USA) in 1997. He served on the ACES Board of Directors from 1998 to 2001 and 2002 to $2003 \mathrm{He}$ served as the ACES Vice President from 1998 to 2001. From 1997 to 1999, he was an Associate Editor of Radio Science. He served as an Associate Editor of the IEEE TRANSACTIONS ON ANTENNAS AND PROPAGATION from 1998 to 2008. 